\title{
The Jensen-Mercer Inequality with Infinite Convex Combinations
}

\author{
Zlatko Pavić*
}

\begin{abstract}
The paper deals with discrete forms of double inequalities related to convex functions of one variable. Infinite convex combinations and sequences of convex combinations are included. The double inequality form of the Jensen-Mercer inequality and its variants are especially studied.

Keywords: double inequality; infinite convex combination; Jensen-Mercer inequality.

AMS Subject Classification (2010): Primary: 26A51 ; Secondary: 26D15.

*Zlatko Pavić
\end{abstract}

\section{Introduction}

The basic framework of convexity includes a bounded closed interval of real numbers, its convex combinations, and corresponding convex function.

Let $a$ and $b$ be real numbers such that $a<b$. The closed interval or segment between points $a$ and $b$ can be introduced as the set

$$
[a, b]=\{\alpha a+\beta b: \alpha, \beta \in[0,1], \alpha+\beta=1\},
$$

that is, as the set of all binomial convex combinations of points $a$ and $b$. In this regard, each point $x \in[a, b]$ can be represented as the binomial convex combination

$$
x=\frac{b-x}{b-a} a+\frac{x-a}{b-a} b,
$$

which shows that $\alpha(x)=(b-x) /(b-a)$ and $\beta(x)=(x-a) /(b-a)$.

A function $f:[a, b] \rightarrow \mathbb{R}$ is said to be convex if the inequality

$$
f(\alpha x+\beta y) \leq \alpha f(x)+\beta f(y)
$$

holds for every binomial convex combination $\alpha x+\beta y$ of points $x, y \in[a, b]$.

\section{Convex Function and its Double Discrete Inequalities}

Generally, a linear combination $\sum_{i=1}^{n} \lambda_{i} x_{i}$ of points $x_{i}$ is said to be convex (affine) if coefficients $\lambda_{i} \in[0,1]$ and $\sum_{i=1}^{n} \lambda_{i}=1\left(\sum_{i=1}^{n} \lambda_{i}=1\right)$.

Let $\sum_{i=1}^{n} \lambda_{i} x_{i}$ be a convex combination of points $x_{i} \in[a, b]$. If $\alpha a+\beta b$ is the convex combination of endpoints $a$ and $b$ such that

$$
\alpha a+\beta b=\sum_{i=1}^{n} \lambda_{i} x_{i}
$$

Received : 01-08-2018, Accepted : 14-03-2019 
then each convex function $f:[a, b] \rightarrow \mathbb{R}$ satisfies the double inequality

$$
f(\alpha a+\beta b) \leq \sum_{i=1}^{n} \lambda_{i} f\left(x_{i}\right) \leq \alpha f(a)+\beta f(b) .
$$

This fundamental inequality says that the convex function values, taken in the forms of convex combinations, grow from the center to the ends. The left-hand side of the above inequality as $f\left(\sum_{i=1}^{n} \lambda_{i} x_{i}\right) \leq \sum_{i=1}^{n} \lambda_{i} f\left(x_{i}\right)$ is the discrete form of Jensen's inequality (see [4]). The proof of the inequality in formula (2.2) can be found in [11, Theorem 3.1].

Using the affine combination $a+b-\sum_{i=1}^{n} \lambda_{i} x_{i}$ and the equality in formula (2.1), each convex function $f$ : $[a, b] \rightarrow \mathbb{R}$ satisfies the double inequality

$$
\begin{aligned}
f\left(a+b-\sum_{i=1}^{n} \lambda_{i} x_{i}\right) & \leq(1-\alpha) f(a)+(1-\beta) f(b) \\
& \leq f(a)+f(b)-\sum_{i=1}^{n} \lambda_{i} f\left(x_{i}\right) .
\end{aligned}
$$

The first and third members represent the Jensen-Mercer inequality obtained in [7], and its generalizations were reached in [6], [8], [3], [10], [5] and others. The proof of the inequality in formula (2.3) can be taken from the proof of Theorem 4.1.

Let $[a, b] \subseteq\left[a_{1}, b_{1}\right]$, let $\sum_{i=1}^{n} \lambda_{i} x_{i}$ be a convex combination of points $x_{i} \in[a, b]$, and let $\sum_{j=1}^{m} \kappa_{j} y_{j}$ be a convex combination of points $y_{j} \in\left[a_{1}, b_{1}\right] \backslash(a, b)$. If the above convex combinations have the same center $c=\alpha a+\beta b$, that is, if

$$
\sum_{i=1}^{n} \lambda_{i} x_{i}=\alpha a+\beta b=\sum_{j=1}^{m} \kappa_{j} y_{j},
$$

then each convex function $f:\left[a_{1}, b_{1}\right] \rightarrow \mathbb{R}$ satisfies the double inequality

$$
\sum_{i=1}^{n} \lambda_{i} f\left(x_{i}\right) \leq \alpha f(a)+\beta f(b) \leq \sum_{j=1}^{m} \kappa_{j} f\left(y_{j}\right) .
$$

The above inequality, even more clearly then the inequality in formula (2.2), shows the nature of growth of the convex function values. The proof of this double inequality can be found in [9, Corollary 3.2].

\section{Infinite Convex Combinations in Inequalities}

In this section, we briefly recall the main results obtained in [12].

Definition 3.1. An infinite linear combination $\sum_{i=1}^{\infty} \lambda_{i} x_{i}$ of a real vector space points $x_{i}$ is said to be convex if coefficients $\lambda_{i} \in[0,1]$ and their sum $\sum_{i=1}^{\infty} \lambda_{i}$ converges to number 1 in the field $\mathbb{R}$. Thus $\sum_{i=1}^{\infty} \lambda_{i}=1$.

Infinite convex combinations are prone to convergence.

Theorem A. An infinite convex combination $\sum_{i=1}^{\infty} \lambda_{i} x_{i}$ of points $x_{i} \in[a, b]$ converges in $[a, b]$.

The inequality in formula (2.2) can be expanded to infinite convex combinations.

Theorem B. Let $\sum_{i=1}^{\infty} \lambda_{i} x_{i}$ be an infinite convex combination of points $x_{i} \in[a, b]$, and let $\alpha a+\beta b$ be the convex combination that satisfies $\alpha a+\beta b=\sum_{i=1}^{\infty} \lambda_{i} x_{i}$.

Then each convex function $f:[a, b] \rightarrow \mathbb{R}$ satisfies the double inequality

$$
f(\alpha a+\beta b) \leq \sum_{i=1}^{\infty} \lambda_{i} f\left(x_{i}\right) \leq \alpha f(a)+\beta f(b) .
$$

The left-hand side of the above inequality as $f\left(\sum_{i=1}^{\infty} \lambda_{i} x_{i}\right) \leq \sum_{i=1}^{\infty} \lambda_{i} f\left(x_{i}\right)$ is Jensen's inequality for infinite convex combinations. 
The inequality in formula (2.5) can also be exposed in the infinite form. Let $[a, b] \subseteq\left[a_{1}, b_{1}\right]$, let $\sum_{i=1}^{\infty} \lambda_{i} x_{i}$ be an infinite convex combination of points $x_{i} \in[a, b]$, and let $\sum_{i=1}^{\infty} \kappa_{i} y_{i}$ be an infinite convex combination of points $y_{i} \in\left[a_{1}, b_{1}\right] \backslash(a, b)$. If the above convex combinations have the same center $c=\alpha a+\beta b$, that is, if

$$
\sum_{i=1}^{\infty} \lambda_{i} x_{i}=\alpha a+\beta b=\sum_{i=1}^{\infty} \kappa_{i} y_{i}
$$

then each convex function $f:\left[a_{1}, b_{1}\right] \rightarrow \mathbb{R}$ satisfies the double inequality

$$
\sum_{i=1}^{\infty} \lambda_{i} f\left(x_{i}\right) \leq \alpha f(a)+\beta f(b) \leq \sum_{i=1}^{\infty} \kappa_{i} f\left(y_{i}\right) .
$$

The double inequality in formula (2.2) can also be expanded to sequences of convex combinations. Because of the possible convergence to the interval endpoints, we have to use a continuous convex function $f$ in Theorem C.

Theorem C. Let $\left(c_{n}\right)_{n=1}^{\infty}$ be a convergent sequence of convex combinations $c_{n}=\sum_{i=1}^{m_{n}} \lambda_{n i} x_{n i}$ of points $x_{n i} \in[a, b]$, and let $\alpha a+\beta b$ be the convex combination that satisfies $\alpha a+\beta b=\lim _{n \rightarrow \infty} c_{n}$.

Then each continuous convex function $f:[a, b] \rightarrow \mathbb{R}$ satisfies the double inequality

$$
f(\alpha a+\beta b) \leq \lim _{n \rightarrow \infty} \sum_{i=1}^{m_{n}} \lambda_{n i} f\left(x_{n i}\right) \leq \alpha f(a)+\beta f(b) .
$$

\section{Main Results}

In this section, we investigate the double inequality form of the Jensen-Mercer inequality (see [7]) including infinite convex combinations.

Lemma 4.1. Let $\sum_{i=1}^{\infty} \lambda_{i} x_{i}$ be an infinite convex combination of points $x_{i} \in[a, b]$. Then the affine combination $a+b-$ $\sum_{i=1}^{\infty} \lambda_{i} x_{i}$ converges in $[a, b]$.

Proof. We can write down $\sum_{i=1}^{\infty} \lambda_{i} x_{i}$ as the convex combination $\alpha a+\beta b$ by Theorem A. Then it follows that

$$
a+b-\sum_{i=1}^{\infty} \lambda_{i} x_{i}=a+b-\alpha a-\beta b=(1-\alpha) a+(1-\beta) b
$$

belongs to $[a, b]$ because the combination $(1-\alpha) a+(1-\beta) b$ is convex.

We have the convenience to expand the inequality in formula (2.3).

Theorem 4.1. Let $\sum_{i=1}^{\infty} \lambda_{i} x_{i}$ be an infinite convex combination of points $x_{i} \in[a, b]$, and let $\alpha a+\beta b$ be the convex combination that satisfies $\alpha a+\beta b=\sum_{i=1}^{\infty} \lambda_{i} x_{i}$.

Then each convex function $f:[a, b] \rightarrow \mathbb{R}$ satisfies the double inequality

$$
\begin{aligned}
f\left(a+b-\sum_{i=1}^{\infty} \lambda_{i} x_{i}\right) & \leq(1-\alpha) f(a)+(1-\beta) f(b) \\
& \leq f(a)+f(b)-\sum_{i=1}^{\infty} \lambda_{i} f\left(x_{i}\right) .
\end{aligned}
$$

Proof. The point $d=a+b-\sum_{i=1}^{\infty} \lambda_{i} x_{i}$ belongs to the interval $[a, b]$ by Lemma 4.1. Applying the convexity of $f$ to the right side of the convex combinations equality

$$
a+b-\sum_{i=1}^{\infty} \lambda_{i} x_{i}=(1-\alpha) a+(1-\beta) b
$$

we get the left-hand side (containing the first and second member) of the inequality in formula (4.1). 
Using the right-hand side (containing the second and third member) of the inequality in formula (3.1) as

$$
-\alpha f(a)-\beta f(b) \leq-\sum_{i=1}^{\infty} \lambda_{i} f\left(x_{i}\right)
$$

we get

$$
\begin{aligned}
(1-\alpha) f(a)+(1-\beta) f(b) & =f(a)+f(b)-\alpha f(a)-\beta f(b) \\
& \leq f(a)+f(b)-\sum_{i=1}^{\infty} \lambda_{i} f\left(x_{i}\right),
\end{aligned}
$$

which is the right-hand side (containing the second and third member) of the inequality in formula (4.1).

The middle member in formula (4.1) can be expressed using endpoints $a$ and $b$, and the combination center $c=\sum_{i=1}^{\infty} \lambda_{i} x_{i}$ as follows.

Corollary 4.1. Let $\sum_{i=1}^{\infty} \lambda_{i} x_{i}$ be an infinite convex combination of points $x_{i} \in[a, b]$, and let $c=\sum_{i=1}^{\infty} \lambda_{i} x_{i}$ be its center.

Then each convex function $f:[a, b] \rightarrow \mathbb{R}$ satisfies the double inequality

$$
\begin{aligned}
f\left(a+b-\sum_{i=1}^{\infty} \lambda_{i} x_{i}\right) & \leq \frac{c-a}{b-a} f(a)+\frac{b-c}{b-a} f(b) \\
& \leq f(a)+f(b)-\sum_{i=1}^{\infty} \lambda_{i} f\left(x_{i}\right) .
\end{aligned}
$$

Proof. Since $c \in[a, b]$, it can be represented as the convex combination

$$
c=\frac{b-c}{b-a} a+\frac{c-a}{b-a} b .
$$

Using the coefficients

$$
1-\alpha=\frac{c-a}{b-a}, \quad 1-\beta=\frac{b-c}{b-a}
$$

in formula (4.1), we obtain formula (4.2).

Theorem 4.1 can be generalized including an $m$-tuple of infinite convex combinations, $\sum_{i=1}^{\infty} \lambda_{i j} x_{i j}$ for $j=$ $1, \ldots, m$.

Corollary 4.2. Let $\sum_{i=1}^{\infty} \lambda_{i j} x_{i j}$ be infinite convex combinations of points $x_{i j} \in[a, b]$, let $\alpha_{j} a+\beta_{j} b$ be the convex combinations that satisfy $\alpha_{j} a+\beta_{j} b=\sum_{i=1}^{\infty} \lambda_{i j} x_{i j}$, and let $\gamma_{j}$ be nonnegative coefficients that satisfy $\sum_{j=1}^{m} \gamma_{j}=1$.

Then each convex function $f:[a, b] \rightarrow \mathbb{R}$ satisfies the double inequality

$$
\begin{aligned}
f\left(a+b-\sum_{j=1}^{m} \sum_{i=1}^{\infty} \gamma_{j} \lambda_{i j} x_{i j}\right) & \leq\left(1-\sum_{j=1}^{m} \alpha_{j} \gamma_{j}\right) f(a)+\left(1-\sum_{j=1}^{m} \beta_{j} \gamma_{j}\right) f(b) \\
& \leq f(a)+f(b)-\sum_{j=1}^{m} \sum_{i=1}^{\infty} \gamma_{j} \lambda_{i j} f\left(x_{i j}\right) .
\end{aligned}
$$

Proof. The points $d_{j}=a+b-\sum_{i=1}^{\infty} \lambda_{i j} x_{i j}$ for $j=1, \ldots, m$ belong to $[a, b]$ by Lemma 4.1. Therefore, the point

$$
\begin{aligned}
d & =a+b-\sum_{j=1}^{m} \sum_{i=1}^{\infty} \gamma_{j} \lambda_{i j} x_{i j}=\sum_{j=1}^{m} \gamma_{j}\left(a+b-\sum_{i=1}^{\infty} \lambda_{i j} x_{i j}\right) \\
& =\sum_{j=1}^{m} \gamma_{j} d_{j}
\end{aligned}
$$

also belongs to $[a, b]$ as the convex combination of points $d_{j} \in[a, b]$. Applying the Jensen inequality to the convex combination

$$
d=\sum_{j=1}^{m} \gamma_{j}\left(a+b-\sum_{i=1}^{\infty} \lambda_{i j} x_{i j}\right),
$$


and using the double inequality in formula (4.1), we obtain the multiple inequality

$$
\begin{aligned}
f(d) & \leq \sum_{j=1}^{m} \gamma_{j} f\left(a+b-\sum_{i=1}^{\infty} \lambda_{i j} x_{i j}\right) \\
& \leq \sum_{j=1}^{m} \gamma_{j}\left(\left(1-\alpha_{j}\right) f(a)+\left(1-\beta_{j}\right) f(b)\right) \\
& \leq \sum_{j=1}^{m} \gamma_{j}\left(f(a)+f(b)-\sum_{i=1}^{\infty} \lambda_{i j} f\left(x_{i j}\right)\right) .
\end{aligned}
$$

Since

$$
\sum_{j=1}^{m} \gamma_{j}\left(\left(1-\alpha_{j}\right) f(a)+\left(1-\beta_{j}\right) f(b)\right)=\left(1-\sum_{j=1}^{m} \alpha_{j} \gamma_{j}\right) f(a)+\left(1-\sum_{j=1}^{m} \beta_{j} \gamma_{j}\right) f(b)
$$

and

$$
\sum_{j=1}^{m} \gamma_{j}\left(f(a)+f(b)-\sum_{i=1}^{\infty} \lambda_{i j} f\left(x_{i j}\right)\right)=f(a)+f(b)-\sum_{j=1}^{m} \sum_{i=1}^{\infty} \gamma_{j} \lambda_{i j} f\left(x_{i j}\right),
$$

the above multiple inequality includes the double inequality in formula (4.3).

Note that the coefficients sum

$$
\left(1-\sum_{j=1}^{m} \alpha_{j} \gamma_{j}\right)+\left(1-\sum_{j=1}^{m} \beta_{j} \gamma_{j}\right)=1 .
$$

In the investigation of means, the Hermite-Hadamard inequality (see [2] and [1]) is almost unavoidable. This important inequality

$$
f\left(\frac{a+b}{2}\right) \leq \frac{1}{b-a} \int_{a}^{b} f(x) d x \leq \frac{f(a)+f(b)}{2}
$$

can also be expressed using the affine combinations $a+b-x$ and $a+b-\sum_{i=1}^{\infty} \lambda_{i} x_{i}$. The midpoint $(a+b) / 2$ dominates again, and it is as follows.

Corollary 4.3. Let $\sum_{i=1}^{\infty} \lambda_{i} x_{i}$ be an infinite convex combination of points $x_{i} \in[a, b]$ such that $(a+b) / 2=\sum_{i=1}^{\infty} \lambda_{i} x_{i}$.

Then each convex function $f:[a, b] \rightarrow \mathbb{R}$ satisfies the double inequality

$$
\begin{aligned}
f\left(a+b-\sum_{i=1}^{\infty} \lambda_{i} x_{i}\right) & \leq \frac{1}{b-a} \int_{a}^{b} f(a+b-x) d x \\
& \leq f(a)+f(b)-\sum_{i=1}^{\infty} \lambda_{i} f\left(x_{i}\right) .
\end{aligned}
$$

Proof. In our case, the midpoint also coincides with the affine combination because

$$
\frac{a+b}{2}=a+b-\sum_{i=1}^{\infty} \lambda_{i} x_{i}
$$

As regards the integral, we have

$$
\int_{a}^{b} f(x) d x=\int_{a}^{b} f(a+b-x) d x
$$

because the mapping $x \mapsto a+b-x$ is a bijection on the interval $[a, b]$.

Applying the right-hand side (containing the second and third member) of the inequality in formula (3.1) to the convex combinations equality

$$
\frac{1}{2} a+\frac{1}{2} b=\sum_{i=1}^{\infty} \lambda_{i} x_{i}
$$


we get

$$
\sum_{i=1}^{\infty} \lambda_{i} f\left(x_{i}\right) \leq \frac{1}{2} f(a)+\frac{1}{2} f(b)=\frac{f(a)+f(b)}{2}
$$

and so

$$
\begin{aligned}
\frac{f(a)+f(b)}{2} & =f(a)+f(b)-\frac{f(a)+f(b)}{2} \\
& \leq f(a)+f(b)-\sum_{i=1}^{\infty} \lambda_{i} f\left(x_{i}\right) .
\end{aligned}
$$

By using the substitutions suggested in the above calculations, formula (4.4) passes into formula (4.5).

Let $[a, b] \subseteq\left[a_{1}, b_{1}\right]$, let $x_{i} \in[a, b]$, and let $y_{i} \in\left[a_{1}, b_{1}\right] \backslash(a, b)$. The double equality

$$
\frac{x_{i}+\left(a+b-x_{i}\right)}{2}=\frac{a+b}{2}=\frac{y_{i}+\left(a+b-y_{i}\right)}{2}
$$

shows that $a+b-x_{i} \in[a, b]$, and that $a+b-y_{i} \in\left[a_{1}, b_{1}\right] \backslash(a, b)$. It follows that

$$
\sum_{i=1}^{\infty} \lambda_{i} x_{i}=\alpha a+\beta b=\sum_{i=1}^{\infty} \kappa_{i} y_{i}
$$

if and only if

$$
\sum_{i=1}^{\infty} \lambda_{i}\left(a+b-x_{i}\right)=(1-\alpha) a+(1-\beta) b=\sum_{i=1}^{\infty} \kappa_{i}\left(a+b-y_{i}\right) .
$$

So, the combinations $\sum_{i=1}^{\infty} \lambda_{i} x_{i}$ and $\sum_{i=1}^{\infty} \kappa_{i} y_{i}$ have the same center if and only if the combinations $\sum_{i=1}^{\infty} \lambda_{i}\left(a+b-x_{i}\right)$ and $\sum_{i=1}^{\infty} \kappa_{i}\left(a+b-y_{i}\right)$ have the same center.

If $\sum_{i=1}^{\infty} \lambda_{i} x_{i}$ is an infinite convex combination of points $x_{i} \in[a, b]$, then using the Jensen inequality for infinite convex combinations, and inequalities

$$
f\left(a+b-x_{i}\right) \leq f(a)+f(b)-f\left(x_{i}\right),
$$

we can prove that a convex function $f:[a, b] \rightarrow \mathbb{R}$ satisfies the double inequality

$$
\begin{aligned}
f\left(a+b-\sum_{i=1}^{\infty} \lambda_{i} x_{i}\right) & \leq \sum_{i=1}^{\infty} \lambda_{i} f\left(a+b-x_{i}\right) \\
& \leq f(a)+f(b)-\sum_{i=1}^{\infty} \lambda_{i} f\left(x_{i}\right) .
\end{aligned}
$$

If $\sum_{i=1}^{\infty} \kappa_{i} y_{i}$ is an infinite convex combination of points $y_{i} \in\left[a_{1}, b_{1}\right] \backslash(a, b)$ such that $\sum_{i=1}^{\infty} \kappa_{i} y_{i} \in\left[a_{1}, b_{1}\right] \backslash(a, b)$, then using the Jensen inequality for infinite convex combinations, and inequalities

$$
f\left(a+b-y_{i}\right) \geq f(a)+f(b)-f\left(y_{i}\right),
$$

we can prove that a convex function $f:\left[a_{1}, b_{1}\right] \rightarrow \mathbb{R}$ satisfies the double inequality

$$
\begin{aligned}
f(a)+f(b)-\sum_{i=1}^{\infty} \kappa_{i} f\left(y_{i}\right) & \leq f\left(a+b-\sum_{i=1}^{\infty} \kappa_{i} y_{i}\right) \\
& \leq \sum_{i=1}^{\infty} \kappa_{i} f\left(a+b-y_{i}\right) .
\end{aligned}
$$

Theorem 4.2. Let $[a, b] \subseteq\left[a_{1}, b_{1}\right]$, let $\sum_{i=1}^{\infty} \lambda_{i} x_{i}$ be an infinite convex combination of points $x_{i} \in[a, b]$, let $\sum_{i=1}^{\infty} \kappa_{i} y_{i}$ be an infinite convex combination of points $y_{i} \in\left[a_{1}, b_{1}\right] \backslash(a, b)$ such that it has the same center as the above, and let $\alpha a+\beta b$ be the convex combination such that $\sum_{i=1}^{\infty} \lambda_{i} x_{i}=\alpha a+\beta b=\sum_{i=1}^{\infty} \kappa_{i} y_{i}$. 
Then each convex function $f:\left[a_{1}, b_{1}\right] \rightarrow \mathbb{R}$ satisfies the following three double inequalities

$$
\begin{aligned}
f(a)+f(b)-\sum_{i=1}^{\infty} \kappa_{i} f\left(y_{i}\right) & \leq(1-\alpha) f(a)+(1-\beta) f(b) \\
\leq & f(a)+f(b)-\sum_{i=1}^{\infty} \lambda_{i} f\left(x_{i}\right) \\
\sum_{i=1}^{\infty} \lambda_{i} f\left(a+b-x_{i}\right) & \leq(1-\alpha) f(a)+(1-\beta) f(b) \\
\leq & \sum_{i=1}^{\infty} \kappa_{i} f\left(a+b-y_{i}\right) \\
\sum_{i=1}^{\infty}\left(\lambda_{i} f\left(a+b-x_{i}\right)-\kappa_{i} f\left(y_{i}\right)\right) & \leq(1-2 \alpha) f(a)+(1-2 \beta) f(b) \\
& \leq \sum_{i=1}^{\infty}\left(\kappa_{i} f\left(a+b-y_{i}\right)-\lambda_{i} f\left(x_{i}\right)\right)
\end{aligned}
$$

Proof. The inequality in formula (4.9) can easily be derived by rearranging the inequality in formula (3.3).

As regards formula (4.10), since $a+b-x_{i} \in[a, b]$ and $a+b-y_{i} \in\left[a_{1}, b_{1}\right] \backslash(a, b)$, we may apply formula (3.3) to the double equality in formula (4.6), and thus obtain formula (4.10).

The inequality in formula (4.11) can be obtained by arranging the sum of the inequalities in formula (4.9) and formula (4.10).

Theorem $C$ can also be adapted to the double inequality form of the Jensen-Mercer inequality. As noted in Section 3 , because of the possible convergence to the interval endpoints, we must use a continuous convex function.

Theorem 4.3. Let $\left(c_{n}\right)_{n=1}^{\infty}$ be a convergent sequence of convex combinations $c_{n}=\sum_{i=1}^{m_{n}} \lambda_{n i} x_{n i}$ of points $x_{n i} \in[a, b]$, and let $\alpha a+\beta b$ be the convex combination that satisfies $\alpha a+\beta b=\lim _{n \rightarrow \infty} c_{n}$.

Then each continuous convex function $f:[a, b] \rightarrow \mathbb{R}$ satisfies the double inequality

$$
\begin{aligned}
f\left(a+b-\lim _{n \rightarrow \infty} \sum_{i=1}^{m_{n}} \lambda_{n i} x_{n i}\right) & \leq(1-\alpha) f(a)+(1-\beta) f(b) \\
& \leq f(a)+f(b)-\lim _{n \rightarrow \infty} \sum_{i=1}^{m_{n}} \lambda_{n i} f\left(x_{n i}\right) .
\end{aligned}
$$

Proof. Applying the inequality in formula (2.3) to the convex combinations equalities

$$
\alpha_{n} a+\beta_{n} b=\sum_{i=1}^{m_{n}} \lambda_{n i} x_{n i},
$$

we get

$$
\begin{aligned}
f\left(a+b-\sum_{i=1}^{m_{n}} \lambda_{n i} x_{n i}\right) & \leq\left(1-\alpha_{n}\right) f(a)+\left(1-\beta_{n}\right) f(b) \\
& \leq f(a)+f(b)-\sum_{i=1}^{m_{n}} \lambda_{n i} f\left(x_{n i}\right) .
\end{aligned}
$$

By letting $n$ tend to infinity, and utilizing the continuity of $f$ via

$$
\lim _{n \rightarrow \infty} f\left(a+b-\sum_{i=1}^{m_{n}} \lambda_{n i} x_{n i}\right)=f\left(a+b-\lim _{n \rightarrow \infty} \sum_{i=1}^{m_{n}} \lambda_{n i} x_{n i}\right),
$$

the above inequality approaches the inequality in formula (4.12). 


\section{Applications to Quasi-Arithmetic Means}

Due to Lemma 4.1, we can consider the quasi-arithmetic means related to infinite convex combinations. Let $\varphi:[a, b] \rightarrow \mathbb{R}$ be a strictly monotone continuous function, let $\left(x_{i}\right)_{i=1}^{\infty}$ be a sequence of points $x_{i} \in[a, b]$, and let $\left(\lambda_{i}\right)_{i=1}^{\infty}$ be a sequence of nonnegative coefficients such that $\sum_{i=1}^{\infty} \lambda_{i}=1$. The discrete quasi-arithmetic mean with the function $\varphi$ of points $x_{i}$ respecting coefficients $\lambda_{i}$ can be defined as the number

$$
M_{\varphi}\left(x_{i} ; \lambda_{i}\right)=\varphi^{-1}\left(\varphi(a)+\varphi(b)-\sum_{i=1}^{\infty} \lambda_{i} \varphi\left(x_{i}\right)\right) .
$$

The number $M_{\varphi}\left(x_{i} ; \lambda_{i}\right)$ belongs to the interval $[a, b]$ because the affine combination $\varphi(a)+\varphi(b)-\sum_{i=1}^{\infty} \lambda_{i} \varphi\left(x_{i}\right)$ converges in the image of $\varphi$ by Lemma 4.1 (the image of $\varphi$ is the bounded closed interval).

The framework of quasi-arithmetic means includes a pair of strictly monotone continuous functions $\varphi, \psi$ : $[a, b] \rightarrow \mathbb{R}$ and the following correlative notion. The function $\psi$ is said to be $\varphi$-convex ( $\varphi$-concave) if the composition $\psi \circ \varphi^{-1}$ is convex (concave). Giving attention to infinite convex combinations, the basic lemma on quasi-arithmetic means applies as follows.

Lemma 5.1. Let $\varphi, \psi:[a, b] \rightarrow \mathbb{R}$ be strictly monotone continuous functions, and let $\sum_{i=1}^{\infty} \lambda_{i} x_{i}$ be an infinite convex combination of points $x_{i} \in[a, b]$.

If either $\psi$ is increasing and $\varphi$-convex or $\psi$ is decreasing and $\varphi$-concave, then

$$
M_{\varphi}\left(x_{i} ; \lambda_{i}\right) \leq M_{\psi}\left(x_{i} ; \lambda_{i}\right) .
$$

If either $\psi$ is decreasing and $\varphi$-convex or $\psi$ is increasing and $\varphi$-concave, then the reverse inequality is valid in formula (5.2).

Proof. We prove the case that $\psi$ is increasing and $\varphi$-convex. Using the inequality of the first and third member in formula (4.1) with the affine combination $\varphi(a)+\varphi(b)-\sum_{i=1}^{\infty} \lambda_{i} \varphi\left(x_{i}\right)$ and the convex function $f=\psi \circ \varphi^{-1}$, we get

$$
\left(\psi \circ \varphi^{-1}\right)\left(\varphi(a)+\varphi(b)-\sum_{i=1}^{\infty} \lambda_{i} \varphi\left(x_{i}\right)\right) \leq \psi(a)+\psi(b)-\sum_{i=1}^{\infty} \lambda_{i} \psi\left(x_{i}\right) .
$$

Acting with the increasing function $\psi^{-1}$ to the above inequality, we obtain the inequality in formula (5.2).

By using the definition in formula (5.1), the power means can be represented by power functions $\varphi(x)=x^{r}$ for $r \neq 0$, and by the limit function in that representation as $r$ approaches 0 . Let $0<a<b$, and let $\sum_{i=1}^{\infty} \lambda_{i} x_{i}$ be an infinite convex combination of points $x_{i} \in[a, b]$. If $r \neq 0$, the power mean with order $r$ of points $x_{i}$ respecting coefficients $\lambda_{i}$ is the expression

$$
M_{r}\left(x_{i} ; \lambda_{i}\right)=\left(a^{r}+b^{r}-\sum_{i=1}^{\infty} \lambda_{i} x_{i}^{r}\right)^{1 / r} .
$$

Letting $r$ tend to 0 , the above expression goes to the indeterminate limit form $1^{\infty}$. As usual, taking the logarithmantilogarithm and using l'Hôpital's rule, we get

$$
\begin{aligned}
M_{0}\left(x_{i} ; \lambda_{i}\right) & =\lim _{r \rightarrow 0} M_{r}\left(x_{i} ; \lambda_{i}\right) \\
& =\exp \left(\ln a+\ln b-\sum_{i=1}^{\infty} \lambda_{i} \ln x_{i}\right) \\
& =a b \prod_{i=1}^{\infty} x_{i}^{-\lambda_{i}} .
\end{aligned}
$$

According to the middle expression on the right side of the above formula, the power mean with order 0 of points $x_{i}$ respecting coefficients $\lambda_{i}$ is represented by the logarithmic function $\varphi(x)=\ln x$ in formula (5.1).

If $r$ and $s$ are real numbers such that $r<s$, then the inequality

$$
M_{r}\left(x_{i} ; \lambda_{i}\right) \leq M_{s}\left(x_{i} ; \lambda_{i}\right)
$$


holds. It can be proved by applying formula (5.2) to each of the following five cases.

The case $r<s<0$. We use the functions $\varphi(x)=x^{r}$ and $\psi(x)=x^{s}$. Since $0<s / r<1$, the function $f(x)=\left(\psi \circ \varphi^{-1}\right)(x)=x^{s / r}$ is concave. Thus $\psi$ is decreasing and $\varphi$-concave.

The case $r<s=0$. We use the functions $\varphi(x)=x^{r}$ and $\psi(x)=\ln x$. Since $1 / r<0$, the function $f(x)=$ $\left(\psi \circ \varphi^{-1}\right)(x)=(1 / r) \ln x$ is convex. Thus $\psi$ is increasing and $\varphi$-convex.

The case $r<0<s$. We use the functions $\varphi(x)=x^{r}$ and $\psi(x)=x^{s}$. Since $s / r<0$, the function $f(x)=$ $\left(\psi \circ \varphi^{-1}\right)(x)=x^{s / r}$ is convex. Thus $\psi$ is increasing and $\varphi$-convex.

The case $0=r<s$. We use the functions $\varphi(x)=\ln x$ and $\psi(x)=x^{s}$. The function $f(x)=\left(\psi \circ \varphi^{-1}\right)(x)=e^{s x}$ is convex. Thus $\psi$ is increasing and $\varphi$-convex.

The case $0<r<s$. We use the functions $\varphi(x)=x^{r}$ and $\psi(x)=x^{s}$. Since $s / r>1$, the function $f(x)=$ $\left(\psi \circ \varphi^{-1}\right)(x)=x^{s / r}$ is convex. Thus $\psi$ is increasing and $\varphi$-convex.

Applying the inequality in formula (5.5) to the harmonic mean $M_{-1}\left(x_{i} ; \lambda_{i}\right)$, geometric mean $M_{0}\left(x_{i} ; \lambda_{i}\right)$ and arithmetic mean $M_{1}\left(x_{i} ; \lambda_{i}\right)$, we gain the version of the best known mean inequality, which includes infinite convex combinations.

Corollary 5.1. Let $0<a<b$, and let $\sum_{i=1}^{\infty} \lambda_{i} x_{i}$ be an infinite convex combination of points $x_{i} \in[a, b]$.

According to the definition in (5.3)-(5.4), the harmonic-geometric-arithmetic mean inequality of points $x_{i}$ respecting coefficients $\lambda_{i}$ stands as

$$
\left(a^{-1}+b^{-1}-\sum_{i=1}^{\infty} \lambda_{i} x_{i}^{-1}\right)^{-1} \leq a b \prod_{i=1}^{\infty} x_{i}^{-\lambda_{i}} \leq a+b-\sum_{i=1}^{\infty} \lambda_{i} x_{i}
$$

\section{References}

[1] Hadamard, J., Étude sur les propriétés des fonctions entières et en particulier d'une fonction considerée par Riemann, J. Math. Pures Appl., 58(1893), 171-215.

[2] Hermite, Ch., Sur deux limites d'une intégrale définie, Mathesis, 3(1883), 82.

[3] Ivelić, S., Matković, A. and Pečarić, J. E., On a Jensen-Mercer operator inequality, Banach J. Math. Anal., 5(2011), 19-28.

[4] Jensen, J. L. W. V., Om konvekse Funktioner og Uligheder mellem Middelværdier, Nyt Tidsskr. Math. B, 16(1905), 49-68.

[5] Khan, M. A., Khan, G. A., Jameel, M., Khan, K. A. and Kilicman, A., New refinements of Jensen-Mercer's inequality J. Comput. Theor. Nanosci., 12(2015), 4442-4449.

[6] Matković, A., Pečarić, J. and Perić, I., A variant of Jensen's inequality of Mercer's type for operators with applications, Linear Algebra Appl., 418(2006), 551-564.

[7] Mercer, A. McD., A variant of Jensen's inequality, JIPAM, 4(2003), Article 73.

[8] Niezgoda, M., A generalization of Mercer's result on convex functions, Nonlinear Anal., 71(2009), 2771-2779.

[9] Pavić, Z., Convex function and its secant, Adv. Inequal. Appl., 2015(2015), Article 5.

[10] Pavić, Z., Generalizations of Jensen-Mercer's inequality, J. Pure Appl. Math. Adv. Appl., 11(2014), 19-36.

[11] Pavić, Z., Geometric and analytic connections of the Jensen and Hermite-Hadamard inequality, Math. Sci. Appl. E-Notes, 4(2016), 69-76.

[12] Pavić, Z., Inequalities with infinite convex combinations, submitted to FILOMAT.

\section{Affiliations}

\section{ZlATKO PAVIĆ}

ADDRESS: Mechanical Engineering Faculty in Slavonski Brod, University of Osijek, Department of Mathematics, 35000, Slavonski Brod-Croatia.

E-MAIL: Zlatko.Pavic@sfsb.hr

ORCID ID: 0000-0003-3512-8947 